\title{
Smokers' acceptance of "cold calls" offering quitline services
}

\section{Amy M Van Deusen, Andrew Hyland, Sara M Abrams, Paula Celestino, Martin C Mahoney, K Michael Cummings}

Objective: A random sample of smokers was offered a transfer to the New York State Smokers' Quitline in order to assess smokers' acceptance of "cold calls" offering quitline services. Method: A 30-minute survey to assess adult tobacco use was administered to 121 western New York smokers who were originally sampled for a random digit dialled survey in 2005, and then re-interviewed one year later, between April and July 2006. Smokers' interest in receiving immediate quitline assistance was assessed at the end of the survey, when the smokers could choose to be transferred to the New York State Smokers' Quitline in order to receive the quitline's cessation services.

Results: $41 \%$ of smokers accepted the offer for, and subsequently received, New York State Smokers' Quitline services. After controlling for factors related to cessation, women were more likely to respond to the offer.

Conclusions: Although telephone quitline utilisation is low, these data suggest that the demand is high and "cold calls" may be a strategy to extend the use of quitline cessation services.

A ccording to the 2005 National Health Interview Survey (NHIS), $42.5 \%$ of smokers reported attempting to quit within the past year; however, most of those who attempted to quit relapsed back to smoking within a short period. ${ }^{1}$ Clinical Practice Guidelines indicate that all smokers making an attempt to quit should receive behavioural counselling and be offered approved stop smoking medication. ${ }^{2-4}$ Despite these recommendations, surveys reveal that the majority of smokers attempt to quit without any assistance whatsoever. ${ }^{5}$ Among those who do attempt to quit, many report using methods with no proved efficacy such as switching to "light" cigarettes. ${ }^{6}$ Population surveys reveal that only about one in four smokers have ever tried an approved stop smoking medication ${ }^{78}$ or received counselling support to quit. ${ }^{5}$

In order for evidence based treatments to make an impact on smoking prevalence, access to and use of evidence based treatments must be enhanced. For example, although telephone quitlines offer the potential to deliver counselling support and access to stop smoking medications to large numbers of smokers, studies report that only a tiny fraction $(<2 \%)$ of smokers have ever contacted a quitline, ${ }^{8}$ even though such services are typically available at no cost. The goal of this pilot study was to assess smokers' willingness to be transferred to the New York State Smokers' Quitline for smoking cessation services after completing a tobacco use survey from a random sample of smokers in western New York State.

\section{METHODS}

A 30-minute survey to assess adult tobacco use was administered to 121 western New York smokers who were originally sampled for a random digit dialled survey in 2005, and then reinterviewed one year later, between April and July 2006.
Among the 428 smokers who completed the 2005 survey, 286 provided contact information and agreed to be re-contacted one year later. Among these, 160 (60\%) completed the survey, 27 $(10 \%)$ refused, and the remainder was lost to follow-up. Respondents who self designated a non-white race were significantly more likely to be lost to follow-up but there were no differences by education, sex, or cigarettes per day. Among the 160 participants who completed the 2006 survey, 141 (88\%) were still smoking at follow-up and $19(12 \%)$ had quit. The question offering smokers the transfer to the quitline was added to the survey after it was already live in the field, so this question was posed to 121 out of 141 smokers. Eligible participants were reminded that they spoke to us a year ago, that this was a follow-up survey to gather information about people's opinions, beliefs, and behaviours regarding tobacco use in western New York, and that the interview covers many topics, including smoking status, exposure to second hand smoke, opinions about tobacco control policies, and health status.

After the 30-minute interview ended, smokers' interest in receiving immediate quitline assistance was assessed with the final question:

“The New York State Smokers' Quitline provides a two week starter kit of nicotine patches for eligible smokers trying to stop smoking. The quitline also provides counselling to help smokers stop smoking, information on available services and treatment, and makes referrals to local programs and clinics. Brochures and recorded information are also available. Would you like me to transfer you?"

The respondent could choose to be transferred to the quitline or to take the quitline number to call at a later time. Respondents were grouped into four categories: (1) those who agreed to be immediately transferred; (2) those who took the number and called the quitline at a later time; (3) those who took the number but never called the quitline; and (4) those who declined both the offer for the transfer and the number. We combined the first two and latter two categories to identify those who received quitline services and those who did not receive quitline services. In addition to descriptive analyses, we constructed a multivariate logistic regression model to identify the characteristics of those who received quitline services. Control variables included sex, race, age, education, cigarettes per day, time to first cigarette after waking, effort to quit in past 12 months, ever called the quitline before, desire to quit, nicotine replacement therapy (NRT) or other cessation pharmacotherapy use on last quit attempt, plan to quit in next 30 days, and likelihood of success if deciding to quit. The survey protocol was developed at Roswell Park Cancer Institute and approved by its institutional review board.

Abbreviations: NHIS, National Health Interview Survey; NRT, nicotine replacement therapy

(a)

(n)

(a)

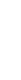


Table 1 Characteristics of smokers who were offered a transfer to the New York State Smokers' quitline for cessation services

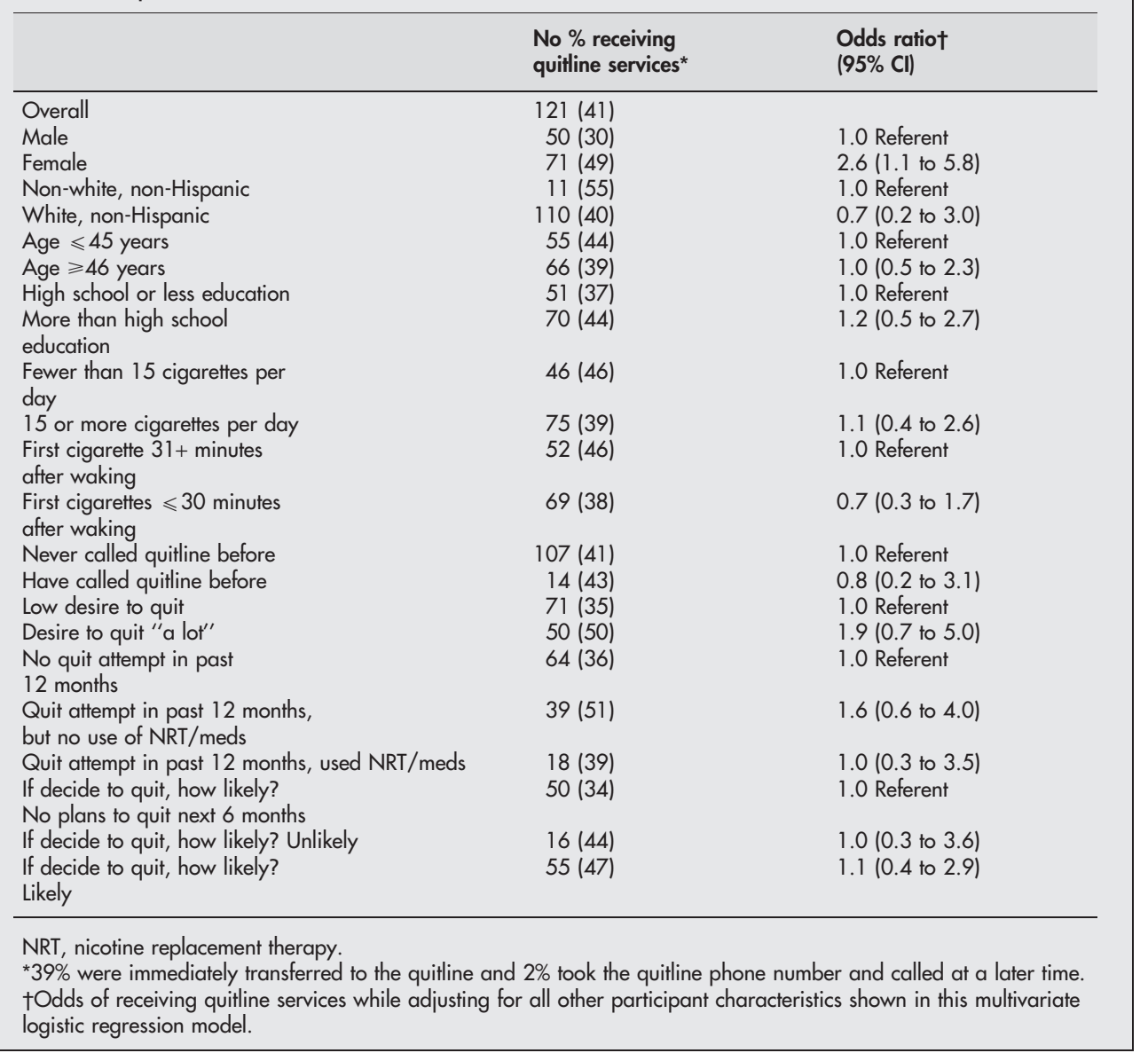

\section{RESULTS}

Among the 121 respondents, $41 \%$ accepted quitline services (39.7\% were immediately transferred and $1.7 \%$ took the number and subsequently called the quitline). Women were more likely to accept immediate service from the quitline when controlling for other factors related to smoking cessation $(\mathrm{OR}=2.6 ; 95 \%$ CI 1.1 to 5.8$)$; there was no difference by any other factors examined.

\section{DISCUSSION}

This study lends support to the idea that a "cold call" approach, proactively offering quitline services, could result in a sizeable increase in utilisation. It is unclear how cost effective "cold calling" may be for encouraging population-wide changes in smoking cessation, but this study does suggest that a large fraction of smokers would immediately utilise evidence based treatment services if offered. It is important to note that the offer of quitline services was posed at the end of the 30-minute survey, by which time some degree of rapport was probably established, and this may have influenced responses. After controlling for other factors related to cessation, women were more likely to accept the transfer offer. While no other significant differences were noted, the limited sample size does not allow for the detection of small differences that may exist.

Consistent with our findings, research done by Paul et al found that over $92 \%$ of smokers in a community based study believe that direct telemarketing of cessation services is acceptable, ${ }^{9}$ suggesting that facilitating referrals to a quitline would have broad appeal to large segments of smokers in the population. For example, healthcare providers and employers could establish systems that would allow them to provide immediate linkage of interested smokers to quitline services. To estimate the population impact of this intervention, one would need to consider not only factors related to accepting the offer once on the phone doing the survey (for example, rapport), but also factors related to their willingness to accept a "cold call." While $41 \%$ accepted the offer for the transfer, it is unknown whether the motivation for the quitline service was for the nicotine replacement therapy, counselling, or some combination of services. Obviously, the findings from this relatively small study need to be repeated and extended to a larger and more diverse sample of smokers to assess how valid and generalisable our conclusions are, the optimal delivery method of the intervention, and its cost effectiveness. However, the

\section{What this paper adds}

Clinical Practice Guidelines indicate that all smokers making an attempt to quit should receive behavioural counselling and be offered approved stop smoking medication, but despite these recommendations, most smokers attempt to quit on their own without any form of assistance. A study in New York State revealed that less than $2 \%$ of smokers have ever contacted a quitline, even though the service is free.

A random sample of 121 smokers was offered a transfer to the New York State Smokers' Quitline at the end of a telephone interview assessing smoking behaviours. Forty-one percent accepted the offer and subsequently received quitline services. This is important because quitline utilisation is low but these data suggest that the demand is high. 
results of this study provide encouraging data suggesting that interest in using quitline services may be much greater than is reflected in current usage rates.

\section{ACKNOWLEDGEMENTS}

This study was funded by the New York State Department of Health.

\section{Authors' affiliations}

Amy M Van Deusen, Andrew Hyland, Sara M Abrams, Paula Celestino, Martin C Mahoney, K Michael Cummings, Roswell Park Cancer Institute, Department of Health Behavior, Buffalo, NY 14263, United States

Correspondence to: K Michael Cummings, PhD, MPH, Roswell Park Cancer Institute, Department of Health Behavior, Elm and Carlton Streets, Buffalo, NY 14263, USA; Michael.Cummings@RoswellPark.org

Received 7 February 2007

Accepted 1 May 2007

\section{REFERENCES}

1 CDC. Tobacco use among adults - United States, 2005. MMWR 2006;55:1145-8.

2 Clinical guidelines for prescribing pharmacotherapy for smoking cessation. US Public Health Service. http://www.surgeongeneral.gov/tobacco/prescrib.htm, accessed 16 January, 2007.

3 Treating tobacco use and dependence. Summary, June 2000. US Public Health Service. http://www.sugeongeneral.gov/tobacco/smokesum.htm. Accessed 6 November, 2006

4 Fiore MC, Bailey WC, Cohen SJ, et al. Treating tobacco use and dependence. quick reference guide for clinicians. Rockville, MD: US Department of Health and Human Services, Public Health Service, October 2000.

5 Zhu S-H, Melcer T, Sun J, et al. Smoking cessation with and without assistance, a population-based analysis. Am J Prev Med 2000;18:305-11.

6 Kozlowski LT, Goldberg ME, Yost BA, et al. Smokers' misperceptions of light and ultra-light cigarettes may keep them smoking. Am J Prev Med 1998;15:9-16.

7 National Cancer Institute. Tobacco use supplement, current population survey. http://riskfactor.cancer.gov/studies/tus-cps/results/data03/. Accessed 16 November, 2006.

8 Farrelly M, Nonnemaker J, Engelen M, et al. Smoking cessation in New York State. Prepared for New York State Department of Health, August 2006

9 Paul CL, Wiggers J, Daly JB, et al. Direct telemarketing of smoking cessation interventions: will smokers take the call? Addiction 2004;99:907-13. 